\title{
Application of contrast-enhanced ultrasound and enhanced CT in diagnosis of liver cancer and evaluation of radiofrequency ablation
}

\author{
SHUHONG KONG $^{1 *}$, XUEWANG YUE $^{1 *}$, SHENG KONG $^{2}$ and YUJIE REN ${ }^{1}$ \\ ${ }^{1}$ Department of Computed Tomography, Dongying People's Hospital, Dongying, Shandong 257091; ${ }^{2}$ Department of \\ Ultrasound, Laiwu Central Hospital of Xinwen Mineral Group, Laiwu, Shandong 271103, P.R. China
}

Received December 7, 2017; Accepted May 4, 2018

DOI: $10.3892 / \mathrm{ol} .2018 .8898$

\begin{abstract}
This study investigated the application effect of contrast-enhanced ultrasound (CEUS) and enhanced $\mathrm{CT}$ in diagnosis of liver cancer and response evaluation of radiofrequency ablation (RFA). A total of 60 patients with liver cancer were selected in Dongying People's Hospital from April 2016 to May 2017. All patients were subjected to CEUS and enhanced CT. With pathological examination as the gold standard, diagnostic consistency of the two methods was compared. After RFA, patients were subjected to CEUS and enhanced CT to assess the efficacy, and the consistency was compared. There was no significant difference in diagnostic accuracy between CEUS and CT ( $p>0.05)$. Area under the ROC curve of CEUS was 0.896, with a sensitivity of $91.2 \%$ and a specificity of $88.7 \%$. The area under the ROC curve for enhanced CT diagnosis was 0.907 , with a sensitivity of $91.8 \%$ and a specificity of $89.7 \%$. No significant difference in the maximal cross sectional area of lesions was found between CEUS and enhanced CT, and there was no significant difference in evaluation of therapeutic efficiency between the methods ( $p>0.05)$ before and 1 and 3 months after treatment. Bland-Altman test showed that there was a strong consistency between CEUS and enhanced CT in the measured maximum cross-sectional area of lesions at 1 and 3 months after treatment. Linear regression analysis showed that maximum section cross-sectional area measured by CEUS was significantly correlated with that detected by enhanced CT $\left(r^{2}=0.617\right)$. The results suggested that diagnostic efficiency of CEUS was similar to that of enhanced CT, and
\end{abstract}

Correspondence to: Dr Sheng Kong, Department of Ultrasound, Laiwu Central Hospital of Xinwen Mineral Group, 3 Youyi North Road, Gangcheng District, Laiwu, Shandong 271103, P.R. China

E-mail: kongsheng6996@163.com

*Contributed equally

Key words: contrast-enhanced ultrasound, enhanced CT, diagnosis of liver cancer, radiofrequency ablation both showed high sensitivity and specificity. Two methods showed high consistency in evaluating the curative effect of RFA. CEUS can achieve real-time observation of focal blood flow perfusion, and was more economically affordable and convenient.

\section{Introduction}

Liver cancer is one of the most common digestive system malignancies in clinical practice. The occurrence and development of liver cancer is a complex process with various factors and pathways involved. With the characteristics of occult onset, fast progress and high mortality, 5-year survival rate of patients with this disease is $<10 \%(1,2)$. Liver cancer mainly affects elderly population, and incidence is higher in men than in women. In recent years, age of onset is becoming younger and younger (3). There are no typical clinical symptoms of early stages of liver cancer, and most patients were diagnosed at advanced stages, so to improve the diagnosis of early liver cancer is particularly important (4). Clinical diagnostic methods for liver cancer include clinical manifestations, laboratory tests and imaging studies. Imaging studies include ultrasound, CT and MRI, within which the diagnosis rate of CT and MRI is high $(5,6)$. With the improvements in contrast-enhanced ultrasound (CEUS), CEUS now can be used to observe blood perfusion of liver tissue in real-time, with high sensitivity and accuracy. Therefore, CEUS has become one of the most commonly used methods for the diagnosis of liver cancer (7). Radiofrequency ablation (RFA) is a non-invasive treatment that has been widely used in clinical practices (8). Enhanced CT can be used to rapidly scan images without blind spots, so it is generally used to evaluate the efficacy of RFA. CEUS can also timely and accurately evaluate the efficacy of RFA (9). In this study, CEUS and enhanced CT were used in the diagnosis of liver cancer and evaluation of efficacy of RFA. Consistency between the two methods was analyzed.

\section{Materials and methods}

General information. A total of 60 patients with liver cancer were selected from April 2016 to May 2017 in Dongying People's Hospital (Dongying, China). All patients were 
Table I. General information of patients.

\begin{tabular}{lc}
\hline Items & Patients $(\mathrm{n}=60)$ \\
\hline Male (n, \%) & $38(63.33)$ \\
Female (n, \%) & $22(36.67)$ \\
Age (years) & $45-79$ \\
Average age (years) & $58.56 \pm 9.43$ \\
Category (n, \%) & \\
Primary & $52(86.67)$ \\
Metastatic & $8(13.33)$ \\
Lesions classification $(\mathrm{n}, \%)$ & \\
Single lesion & $55(91.67)$ \\
Multiple lesions & $5(8.33)$ \\
\hline
\end{tabular}

subjected to CEUS and enhanced CT, and finally diagnosed by pathological examination. All patients underwent EFA, and CEUS and CT were used to evaluate curative effect. The general information of these patients are presented in Table I. The study was approved by the Ethics Committee of Dongying People's Hospital (Dongying, China). Signed informed consents were obtained from the patients.

\section{Method}

Preparation before examination. Patients were fasted for $6 \mathrm{~h}$ before CT scan. All patients were informed with all details in the whole procedure. Breathing training (breathe slowly) was performed to avoid the effects of changes in respiratory rate on image quality.

Enhanced CT examination. Patients were fixed in supine position and both hands were placed on a headrest. Dual Source CT scanner (Siemens AG, Munich, Germany) was used for scanning. Non-ionic iodine contrast agent iohexol $(300 \mathrm{mg} \mathrm{I} / \mathrm{ml}$; Bayer Schering Pharma AG, Berlin, Germany) was injected $(3.0 \mathrm{ml} / \mathrm{sec})$ into the elbow vein of patients as a dose of $1.5 \mathrm{ml} / \mathrm{kg}$. Dynamic enhanced scanning was performed at sub-arterial phase (20-30 sec after injection of iohexol), intravenous phase (60-70 sec after injection of iohexol) and balance phase (150-240 sec after injection of iohexol). All images were sent to the workstation.

CEUS. CEUS was performed using Philips iU22 color Doppler system (Philips Healthcare, Amsterdam, The Netherlands). Probe frequency, 3.0 MHz; harmonic frequency, 3.5 MHz; speed range, 0.06-0.12 m/sec. Contrast agent SonoVue (registration no. H20080059; Bracco, Milan, Italy) was used. One dose of SonoVue was mixed with $5 \mathrm{ml}$ of $9 \%$ sodium chloride solution to make a suspension (density of sulfur hexafluoride microbubbles was $2 \times 10^{8} / \mathrm{ml}$ ). Ultrasonic probe section was fixed in the target area. Contrast agent was injected into peripheral vein. When contrast agent reached the target, lesions were scanned through fan-shaped approach, and contrast agent enhancement and perfusion were timely observed.

Treatment. After local anesthesia, cool-tip RFA needles were inserted through the marked skin. The number of needles was determined by the size of the lesion: 2 needles were used if
Table II. Comparison of the accuracy of CEUS and enhanced CT for the diagnosis of liver cancer $(n, \%)$.

\begin{tabular}{lcccc}
\hline Methods & $\begin{array}{c}\text { Confirmed } \\
\text { diagnosis }\end{array}$ & Misdiagnosis & $\begin{array}{c}\text { Erroneous } \\
\text { diagnosis }\end{array}$ & $\begin{array}{c}\text { Diagnostic } \\
\text { accuracy }\end{array}$ \\
\hline CEUS & $53(88.33)$ & $3(5.00)$ & $4(6.67)$ & $53(88.33)$ \\
Enhanced CT & $55(91.67)$ & $2(3.33)$ & $3(5.00)$ & $55(91.67)$ \\
$\chi^{2}$ & & & & 0.093 \\
P-value & & & & 0.761 \\
\hline
\end{tabular}

CEUS, contrast-enhanced ultrasound.

the tumor diameter was $>3.0 \mathrm{~cm}$, otherwise only 1 needle was used. LDRF-120S multipole RFA instrument (Mianyang Lide Electronic Technology Co., Ltd., Mianyang, China) was used to treat the lesion after the tip of the needle reached the bottom of the tumor. Treatment was monitored under ultrasound, and the whole process was between 10 and $20 \mathrm{~min}$.

Evaluation method. Examination results were analyzed by two senior diagnostic imaging physicians with $>10$ years of work experience using double-blind method. Another imaging physician was included in case of inconsistence. With pathological examination results as gold standard, accuracies of CEUS and enhanced CT were compared. Maximum section area was measured by CEUS and enhanced CT at 1 and 3 months after treatment. Measurement was performed 3 times and the average value was calculated. Cross-sectional area was calculated according to the following formula: $S=1 / 4 \pi X Y$ (X, left and right diameter; $\mathrm{Y}$, front and back diameter).

Evaluation standard of RFA efficacy was as follows (10): i) Completely effective: Fibroid volume reduction rate of $\geq 50 \%$; ii) partially effective: Fibroid volume reduction rate of $<50 \%$; and iii) invalid: Fibroid recurrence or fibroid volume increased. Fibroid volume reduction rate $=$ (fibroid volume before treatment - fibroid volume after treatment) / fibroid volume before treatment $\times 100 \%$. Treatment effective rate $=$ partially effective rate + partial effective rate.

Statistical analysis. Statistical software SPSS 19.0 (SPSS, Inc., Chicago, IL, USA) was used. Measurement data were expressed as mean \pm standard deviation (mean \pm SD) and processed using t-test. Enumeration data were expressed as number or (\%) and processed using $\chi^{2}$ test. ANOVA was used for comparison between multiple groups and the post hoc test was Dunnett's test. Consistency of efficacy evaluation was analyzed using Bland-Altman test. Correlation between the two different examination methods was analyzed by Linear regression analysis, and the closer the value of $\mathrm{r}^{2}$ was to 1 , the higher the correlation value between the two methods was. Area under the ROC curve, and sensitivity and specificity of two methods were analyzed, and the significance level of test was $\alpha=0.05$.

\section{Results}

Comparison of the accuracy of two methods for the diagnosis of liver cancer. No significant differences in the accuracy of 
Table III. Comparison of the maximum tumor cross-sectional area measured by two methods $\left(\operatorname{mean} \pm \mathrm{SD}, \mathrm{cm}^{2}\right)$.

\begin{tabular}{lcccc}
\hline Methods & Cases & $\begin{array}{c}\text { Before } \\
\text { treatment }\end{array}$ & $\begin{array}{c}1 \text { month after } \\
\text { treatment }\end{array}$ & $\begin{array}{c}3 \text { months after } \\
\text { treatment }\end{array}$ \\
\hline CEUS & 60 & $5.56 \pm 1.36$ & $3.47 \pm 1.07$ & $1.15 \pm 0.52$ \\
Enhanced CT & 60 & $5.68 \pm 1.74$ & $3.51 \pm 1.09$ & $1.13 \pm 0.54$ \\
t-test & & 0.421 & 0.203 & 0.517 \\
P-value & & 0.675 & 0.839 & 0.606 \\
\hline
\end{tabular}

CEUS, contrast-enhanced ultrasound.

Table IV. Comparison of the evaluation of RFA efficacy by two methods (n, \%).

\begin{tabular}{lccc}
\hline Methods & Cases & $\begin{array}{c}1 \text { month after } \\
\text { treatment }\end{array}$ & $\begin{array}{c}3 \text { months after } \\
\text { treatment }\end{array}$ \\
\hline CEUS & 60 & $49(81.67)$ & $56(93.33)$ \\
Enhanced CT & 60 & $47(78.33)$ & $59(98.33)$ \\
t-test & & 0.052 & 0.835 \\
P-value & & 0.819 & 0.361 \\
\hline
\end{tabular}

RFA, radiofrequency ablation; CEUS, contrast-enhanced ultrasound.

the two methods for the diagnosis of liver cancer were found ( $>0.05$; Table II).

Comparison of area under the ROC curve of CEUS and enhanced CT. Area under the ROC curve of CEUS was 0.896 with a sensitivity of $90.2 \%$ and a specificity of $88.7 \%$. The area under the ROC curve for enhanced CT diagnosis was 0.907 with a sensitivity of $91.8 \%$ and a specificity of $89.7 \%$. Detailed results are presented in Fig. 1.

Comparison of maximum tumor cross-sectional area measured by two methods. There was no significant difference in maximum tumor cross-sectional area measured by the two methods at 1 and 3 months after treatment $(\mathrm{p}>0.05)$. As shown in Table III.

Comparison of the evaluation of RFA efficacy by two methods. No significant differences were found in evaluation of RFA efficacy by two methods at 1 and 3 months after treatment $(\mathrm{p}>0.05)$. As shown in Table IV.

Analysis of the consistency of CEUS and enhanced CT in measuring maximum tumor cross-sectional area. Bland-Altman test showed that, at 1 month after treatment, as can be seen from Fig. 2, the mean of the difference between the paired data of 60 patients was $0.8 \mathrm{~cm}^{2}$, and the $95 \%$ agreement margin was 3.8 to $-2.3 \mathrm{~cm}^{2}$, and $8.33 \%(5 / 60)$ points are outside the $95 \%$ consistency limit. At 3 months after treatment, as can be seen from Fig. 3, the mean of the paired data differences for the 60 patients was $0.3 \mathrm{~cm}^{2}$, and the $95 \%$ agreement margin

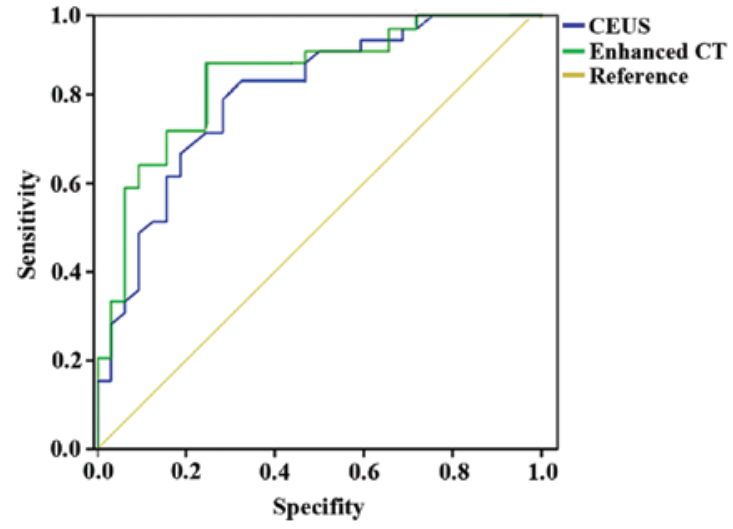

Figure 1. ROC curves of two methods. ROC curve was used to detect the sensitivity and specificity of two methods. No significant differences in the accuracy of the two methods for the diagnosis of liver cancer were found ( $>0.05)$. CEUS, contrast-enhanced ultrasound.

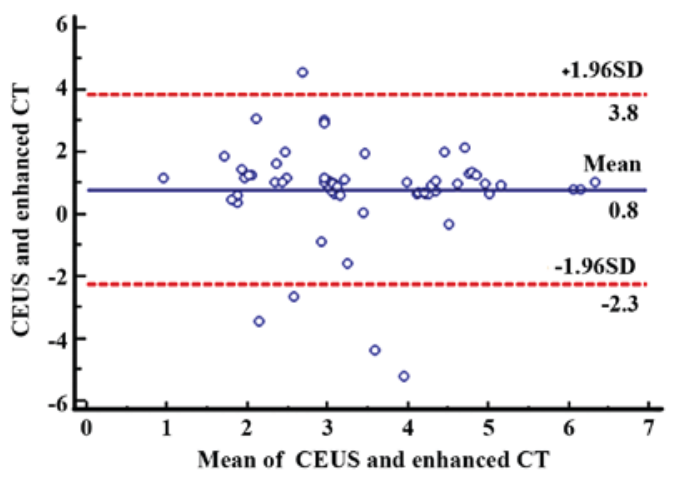

Figure 2. BA map of the differences of CEUS and enhanced CT at 1 month after treatment. Bland-Altman test was used to measure maximum tumor cross-sectional area at 1 month after treatment, the mean of the difference between the paired data of 60 patients was $0.8 \mathrm{~cm}^{2}$, and the $95 \%$ agreement margin was 3.8 to $-2.3 \mathrm{~cm}^{2}$, and $8.33 \%(5 / 60)$ points are outside the $95 \%$ consistency limit. CEUS, contrast-enhanced ultrasound.



Figure 3. BA map of the differences of CEUS and enhanced CT at 3 months after treatment. Bland-Altman test was used to measure maximum tumor cross-sectional area at 3 month after treatment, the mean of the paired data differences for the 60 patients was $0.3 \mathrm{~cm}^{2}$, and the $95 \%$ agreement margin was 2.2 to $-1.6 \mathrm{~cm}^{2}$, and $5 \%(3 / 60)$ points were outside the $95 \%$ agreement limits. CEUS, contrast-enhanced ultrasound.

was 2.2 to $-1.6 \mathrm{~cm}^{2}$, and $5 \%(3 / 60)$ points were outside the $95 \%$ agreement limits, indicating a strong agreement between the two methods. 


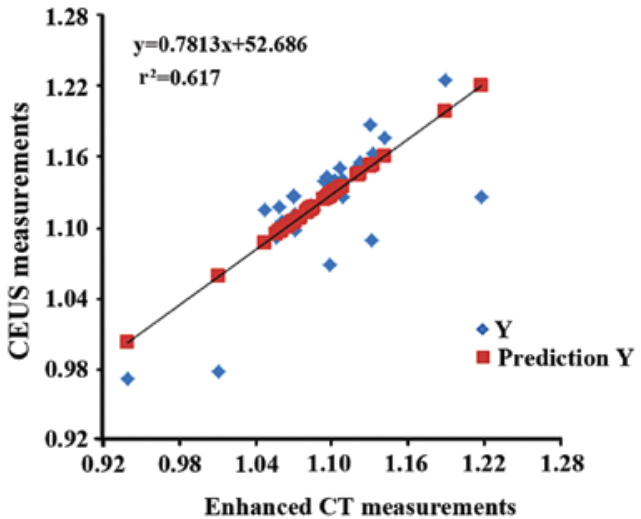

Figure 4. Pearson's correlation analysis of CEUS measurements and enhanced CT measurements. Pearson's correlation analysis was used to evaluate the relationship between CEUS and enhanced CT. Linear regression analysis equation was: $\mathrm{Y}=0.7813 \mathrm{X}+52.686(\mathrm{Y}$, CEUS measurement; $\mathrm{X}$, enhanced CT measurement). CEUS, contrast-enhanced ultrasound.

Correlation analysis of CEUS and enhanced CT in measuring maximum cross-sectional area after treatment. Pearson's correlation analysis equation was: $\mathrm{Y}=0.7813 \mathrm{X}+52.686$ (Y, CEUS measurement; X, enhanced CT measurement). Pearson's correlation analysis showed a strong correlation between CEUS measurements and enhanced CT measurements $\left(r^{2}=0.617\right.$, Fig. 4$)$.

\section{Discussion}

Liver cancer often occurs in the intrahepatic bile duct or liver cells, of which the former can account for $>80 \%$ of the cases, and its incidence and mortality ranks in the forefront of all malignancies (11). Clinical manifestations of liver cancer patients include loss of appetite, liver pain, weight loss, jaundice and ascites, and even coma and systemic failure (12). Liver cancer can be caused by a variety of factors including viral infection, cirrhosis, alcohol and tobacco consumption, chemical carcinogens, drinking water pollution and genetic factors (13). Pathogenesis of liver cancer is complex, and its occurrence is usually the result of long-term cumulative changes in multiple factors. Interaction between environmental factors and genetic polymorphisms plays an important role in the occurrence and development of liver cancer (14). Attention should be paid to the regular screening of liver cancer to achieve early detection, and early diagnosis and early treatment are of great significance to improve the prognosis and reduce the mortality of liver cancer.

With the rapid development of medical imaging technology, it plays an important role in detecting, characterizing, locating and staging liver cancer. Its diagnostic value far exceeds that of serology and has been widely used clinically. CT scanning has the characteristics of fast scanning, clear image and minimal influence from the surrounding organs. With the emergence of multi-slice CT, scanning time becomes even shorter, motion artifact interference is even less, Z-axis resolution and the diagnostic accuracy are even higher (15). Enhanced CT scans through iohexol can not only effectively detect liver cancer lesions, but also improve the accuracy of its qualitative judgment, which in turn increase liver cancer detection rate (16).
Results of this study showed that, compared with the results of pathological diagnosis, the accuracy of enhanced CT in the diagnosis of liver cancer was $91.67 \%$, area under the ROC curve was 0.907 , with a sensitivity of $91.8 \%$ and a specificity of 89.7\%. Diagnostic accuracy of CEUS was $91.67 \%$. Area under ROC curve was 0.896 , the sensitivity was $90.2 \%$ and specificity was $88.7 \%$. No significant differences in diagnostic accuracy was found between the two methods ( $p>0.05$ ), and diagnostic accuracy of both methods was high. This is because enhanced scan in three phases performed according to the three kinds of blood supply characteristics of liver cancer (hepatic artery blood supply, double hepatic artery and portal vein blood supply to portal vein-based hepatic artery less blood supply) can obtain data of arterial, stable and delayed phases of liver tissue and get information on the internal tumor hemodynamic changes, which can accurately reflect the characteristics of the blood supply, so as to improve the accuracy of diagnosis of liver cancer (17). With the application of contrast agent, diagnostic level of ultrasound is improved. CEUS can objectively display the shape, size and number of tumors, and at the same time, it can reflect the characteristics of blood flow inside the tumor (18), which is similar to the function of enhanced CT. CEUS can be an alternative to enhanced CT in the diagnosis of liver cancer, and can provide a reliable basis for treatment of liver cancer.

Treatment of liver cancer with RFA is easy and repeatable without radioactive injury. The principle is: High frequency AC electromagnetic waves generate biological energy to heat the lesions to reach $43-60^{\circ} \mathrm{C}$, so as to cause tumor cell protein denaturation and tissue coagulation necrosis. At the same time, it can also cause coagulation reaction around the tumor, which in turn inhibits tumor metastasis without inducing significant damage to surrounding healthy tissues (19). After treatment, the curative effect needs to be evaluated to determine whether it is necessary to repeat the treatment. Clinical evaluation of RFA is usually performed by imaging examination, which can include ultrasound, CT and MRI (20). The results of this study showed that there was no difference in evaluating efficacy of RFA between CEUS and enhanced $\mathrm{CT}$, and results of Bland-Altman test showed that there was a strong consistency between CEUS and CT. Linear regression analysis showed a strong linear correlation between the two methods $\left(r^{2}=0.617\right)$. The data suggest that CEUS can completely reflect the degree of tumor necrosis after RFA treatment, so as to avoid residual tumor recurrence after RFA due to inaccurate assessment, and its evaluation efficiency is comparable to that of enhanced CT. Clinical application of CT has a certain degree of radioactive damage and imaging section is fixed, while CEUS has the characteristics of convenient and rapid operation, non-radiation, non-space and time constraints, and high repeatability. So its clinical value is higher than that of CEUS.

In summary, CEUS and enhanced CT have high accuracy and consistency in diagnosis if liver cancer and evaluation of RFA efficacy, they can both provide a reliable basis for the diagnosis and treatment of liver cancer.

\section{Acknowledgements}

Not applicable. 


\section{Funding}

No funding was received.

\section{Availability of data and materials}

The datasets used and/or analyzed during the present study are available from the corresponding author on reasonable request.

\section{Authors' contributions}

ShuhongK and XY conceived and designed the study. ShengK and YR were responsible for the collection, analysis and interpretation of the data. ShuhongK drafted the manuscript. YR revised the manuscript critically for important intellectual content. All authors read and approved the final manuscript.

\section{Ethics approval and consent to participate}

The study was approved by the Ethics Committee of Dongying People's Hospital (Dongying, China). Signed informed consents were obtained from the patients.

\section{Patient consent for publication}

Not applicable.

\section{Competing interests}

The authors declare that they have no competing interests.

\section{References}

1. Younossi ZM, Otgonsuren M, Henry L, Venkatesan C, Mishra A, Erario $\mathrm{M}$ and Hunt $\mathrm{S}$ : Association of nonalcoholic fatty liver disease (NAFLD) with hepatocellular carcinoma (HCC) in the United States from 2004 to 2009. Hepatology 62: 1723-1730, 2015.

2. Lee JM,Park JW and Choi BI: 2014 KLCSG-NCC Korea Practice Guidelines for the management of hepatocellular carcinoma: HCC diagnostic algorithm. Dig Dis 32: 764-777, 2014.

3. Kew MC: Hepatocellular carcinoma: Epidemiology and risk factors. J Hepatocell Carcinoma 1: 115-125, 2014.

4. Hann HW, Coben R, Brown D, Needleman L, Rosato E, Min A, Hann RS, Park KB, Dunn S and DiMarino AJ: A long-term study of the effects of antiviral therapy on survival of patients with HBV-associated hepatocellular carcinoma (HCC) following local tumor ablation. Cancer Med 3: 390-396, 2014.

5. Vardal J, Salo RA, Larsson C, Dale AM, Holland D, Groote IR and Bjørnerud A: Correction of B0-distortions in echo-planar-imaging-based perfusion-weighted MRI. J Magn Reson Imaging 39: 722-728, 2014

6. Yu MH, Kim JH, Yoon JH, Kim HC, Chung JW, Han JK and Choi BI: Small $(\leq 1-\mathrm{cm})$ hepatocellular carcinoma: Diagnostic performance and imaging features at gadoxetic acid-enhanced MR imaging. Radiology 271: 748-760, 2014.
7. Jiang N, Xie B, Zhang X, He M, Li K, Bai J, Wang Z, He J and Zhang L: Enhancing ablation effects of a microbubble-enhancing contrast agent ('SonoVue') in the treatment of uterine fibroids with high-intensity focused ultrasound: A randomized controlled trial. Cardiovasc Intervent Radiol 37: 1321-1328, 2014.

8. Bends R, Brössner A, Felberbaum R and Römer T: Myoma in statu nascendi after transcervical radiofrequency ablation of a transmural uterine leiomyoma. Gynakologische Endokrinologie 14: 291-294, 2016 (In German)

9. Wang W, Liu JY, Yang Z, Wang YF, Shen SL, Yi FL, Huang Y, Xu EJ, Xie XY, Lu MD, et al: Hepatocellular adenoma: Comparison between real-time contrast-enhanced ultrasound and dynamic computed tomography. Springerplus 5: 951, 2016.

10. Sung JY, Baek JH, Jung SL, Kim JH, Kim KS, Lee D, Kim WB and Na DG: Radiofrequency ablation for autonomously functioning thyroid nodules: A multicenter study. Thyroid 25: 112-117, 2015.

11. Ikai I,Arii S,Okazaki M,Okita K,Omata M,Kojiro M,Takayasu K, Nakanuma Y, Makuuchi M, Matsuyama Y, et al: Report of the 17th nationwide follow-up survey of primary liver cancer in japan. Hepatol Res 37: 676-691, 2007.

12. Hung HH, Chao Y, Chiou YY, Li CP, Lee RC, Huo TI, Huang YH, Chau GY, Su CW, Yeh YC, et al: A comparison of clinical manifestations and prognoses between patients with hepatocellular carcinoma and Child-Pugh scores of 5 or 6 . Medicine (Baltimore) 93: e348, 2014.

13. Liu F, Wang J, Chang H, Lu J and Li H: Relevance between HLA-DP gene rs2281388 polymorphism and hepatocellular carcinoma risk. Int J Clin Exp Pathol 8: 7431-7435, 2015.

14. Hong YM, Yoon KT, Cho M, Heo J, Woo HY and Lim W: A case of small hepatocellular carcinoma with an extensive lymph node metastasis at diagnosis. Clin Mol Hepatol 20: 310-312, 2014.

15. Chou CT, Chen RC, Lin WC, Ko CJ, Chen CB and Chen YL: Prediction of microvascular invasion of hepatocellular carcinoma: Preoperative CT and histopathologic correlation. AJR Am J Roentgenol 203: W253-W259, 2014.

16. George RT, Mehra VC, Chen MY, Kitagawa K, Arbab-Zadeh A, Miller JM, Matheson MB, Vavere AL, Kofoed KF, Rochitte CE, et al: Myocardial CT perfusion imaging and SPECT for the diagnosis of coronary artery disease: A head-to-head comparison from the CORE320 multicenter diagnostic performance study. Radiology 272: 407-416, 2014.

17. Liu J-J, Li H-X, Chen Z-B, Yang W-P, Zhao S-F, Chen J, Bai T, $\mathrm{Li} \mathrm{H}$ and Li L-Q: Consistency analysis of contrast-enhanced ultrasound and contrast-enhanced CT in diagnosis of small hepatocellular carcinoma. Int J Clin Exp Med 8: 21466-21471, 2015.

18. Trenker C, Neesse A and Görg C: Sonographic patterns of renal lymphoma in B-mode imaging and in contrast-enhanced ultrasound (CEUS) - a retrospective evaluation. Eur J Radiol 84: 807-810, 2015.

19. Zhang ZG, Zhang WG, Wu YH, Liang HF, Zhang BX and Chen XP: Incomplete RFA-generated heat shock response provokes colorectal cancer liver metastases (CRLMs) recurrence by inducing cancer cell stemness and invasion. Mol Cancer Res 13 (10 Suppl): Abst B47, 2015.

20. Bo XW, Xu HX, Sun LP, Zheng SG, Guo LH, Lu F, Wu J and Xu XH: Bipolar radiofrequency ablation for liver tumors: Comparison of contrast-enhanced ultrasound with contrastenhanced MRI/CT in the posttreatment imaging evaluation. Int J Clin Exp Pathol 7: 6108-6116, 2014.

This work is licensed under a Creative Commons Attribution-NonCommercial-NoDerivatives 4.0 International (CC BY-NC-ND 4.0) License. 